\title{
Slow dynamics of the contact process on complex networks
}

\author{
Géza Ódor ${ }^{1, a}$ \\ ${ }^{1}$ Research Centre for Natural Sciences, Hungarian Academy of Sciences, MTA TTK MFA, P. O. Box 49, \\ H-1525 Budapest, Hungary
}

\begin{abstract}
Résumé. The Contact Process has been studied on complex networks exhibiting different kinds of quenched disorder. Numerical evidence is found for Griffiths phases and other rare region effects, in Erdős Rényi networks, leading rather generically to anomalously slow (algebraic, logarithmic,...) relaxation. More surprisingly, it turns out that Griffiths phases can also emerge in the absence of quenched disorder, as a consequence of sole topological heterogeneity in networks with finite topological dimension. In case of scalefree networks, exhibiting infinite topological dimension, slow dynamics can be observed on tree-like structures and a superimposed weight pattern. In the infinite size limit the correlated subspaces of vertices seem to cause a smeared phase transition. These results have a broad spectrum of implications for propagation phenomena and other dynamical process on networks and are relevant for the analysis of both models and empirical data.
\end{abstract}

\section{Introduction}

Nonequilibrium systems have been a central research topic of statistical mechanics [1-3]. As in equilibrium they exhibit various phase transitions, critical phenomena and universality classes. Since they cannot be treated by thermodynamical formalism universality provides a guidance's in the zoo of models exhibiting diverging correlation length and scaling behavior [4]. Power-law scaling behavior has been reported in many real and model systems for spontaneous occurrence the theory of self-organizing-criticality was proposed [5]. This, however requires the competition of a slow accumulation and fast dissipation mechanism. Diverging correlation length on the other hand is generated naturally in nonequilibrium systems by the currents or fields acting on them. Furthermore, quenched disorder may also cause extended regions in the parameter space with non-universal power-law dynamics [44]. A fundamental dynamical system model is the Contact Process (CP) [6, 7], in which sites can be either occupied (infected) or empty (susceptible). By changing the infection rate of the neighbors $\lambda / k$, where $k$ is the degree of the vertex, a continuous phase transition occurs at the $\lambda_{c}$ critical point from inactive to active steady state. The inactive state, characterized by the order parameter, the density of infection $(\rho)$ is zero. This is also called absorbing state, because no spontaneous activation of sites is allowed.

In the new century the interest is shifting from models, defined on Euclidean, regular lattices to processes defined on general networks $[8,9]$. Since the introduction of a simple model describing the emergence of scaling in random networks [10] the study of complex networks is flourishing.

a. e-mail: odor@mfa.kfki.hu

This is an Open Access article distributed under the terms of the Creative Commons Attribution License 2.0, which permits unrestricted use, distribution, and reproduction in any medium, provided the original work is properly cited. 
Multidisciplinary applications involve, for example, the WWW, various biological, sociological and technological networks. These are, mostly scale-free (SF) networks, exhibiting $P(k) \sim k^{-\gamma}$ degree distribution of the nodes (for recent reviews see [8],[11]). Other families of complex network models are those composed of a $d$-dimensional regular lattice and additional long edges [12]. These arise in the context of conductive properties of linear polymers with cross-links that connect remote monomers [13], in public traffic systems [14], in the case of nanowires [15], in social [16] and phone networks [17] or to describe forest fires [18] among other examples. In these models a pair of nodes separated by the distance $l$ of the base lattice are re-connected by a long edge with the asymptotic probability for large $l$ :

$$
p(l)=\beta l^{-s} .
$$

In the special case $s=0$, edges exist with a length-independent probability, as in small world networks, therefore these models are called Generalized Small World networks (GSW). The $s=2$ case is important from network optimization point of view [14] for example. If $s \geq 2$, they are characterized by a finite topological dimension $d$, i.e. $N(l) \sim l^{d}$, where $N(l)$ is the number of nodes within the graph distance $l$ from a given node. Note that if the links vary quickly with respect the time scale of the dynamical process defined on these networks they realize Lévy flights [20, 21]. Usually these annealed networks exhibit different behavior than the quenched ones.

Although many network models exhibit infinite topological dimension $(d)$, simple mean-field approximations cannot capture several important features [23-28]. Very recently it has been conjectured [29-31] that generic slow (power-law, or logarithmic) dynamics is observable only in networks with finite $d$. This claim is relevant in the light of recent developments of dynamical processes on complex networks such as the simple model of "working memory" [32], brain dynamics [33], social networks with heterogeneous communities [34], or to understand the slow relaxation in glassy systems [35]. Slow dynamics has been shown to originate from the bursty behavior of the agents connected by small world networks resulting in memory effects [42]. On the other hand it can also be related to arbitrarily large $(l<N)$, correlated rare-regions (RR), which possess long lifetime in the inactive phase, above the pure critical point $\lambda_{c}^{0}<\lambda<\lambda_{c}$. This can be understood by non-perturbative methods [36-41].

More recently the possibility of power-law dynamics of CP has been investigated on different BA networks with $\gamma=3[28,45]$. Extensive simulations showed $\rho(t) \propto 1 /(t \ln (t))$ density decay and $\rho(\lambda, t \rightarrow \infty) \propto|\lambda-1|$ steady state behavior with logarithmic corrections in agreement with the HMF approximations. On loop-less BA trees the epidemic propagation slows down and a nontrivial critical density decay emerges $\rho\left(t, \lambda_{c}\right) \propto t^{-0.5}$. Additionally, when $k$ dependent weighting was applied, which suppress hubs or make the network disassortatative GP-like dynamics was observed in the simulations. However, systematic finite scaling study revealed that these power-laws saturate in the $N \rightarrow \infty$ thermodynamic limit, suggesting smeared phase transitions known from Euclidean, regular systems if the correlated subspaces can undergo phase transitions themselves, when they are effectively above the lower critical dimension of the problem : $d_{R R}>d_{c}^{-}$[44]. In this case, the dynamics of the locally ordered RR-s completely freezes, and they develop a truly static order parameter. Clearly in infinite dimensional networks such RR-s can be embedded as a percolation analysis confirmed this [45].

\section{Optimal fluctuation theory}

The basic idea of the optimal fluctuation theory is that the long-time decay of the order parameter $(\rho(t))$ is dominated by the regions of size $l$, which are rare in general : $P(l) \propto \exp (-c l)$, but can exhibit exponentially long lifetimes $\tau(l) \propto \exp (b l)$. In particular for the the density of infected sites of CP, 


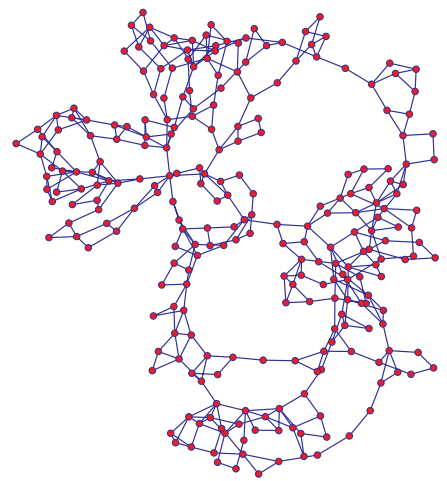

(a)

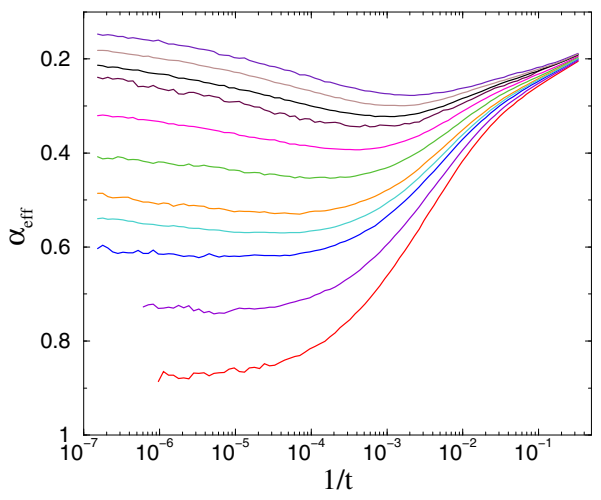

(b)

Figure 1: (a) Topological view of a marginal GSW network [46] with parameters $\beta=1$ and $N=256$ nodes. (b) Local slopes of the density decay in such networks with $\beta=0.2$ and $N=10^{5}$ nodes.

RR-s provide the leading order contribution in the inactive phase :

$$
\rho(t) \sim \int l P(l) \exp (-t / \tau) d l
$$

which in the saddle point approximation results in $\rho(t) \sim t^{-c / b}$ decay, with a non-universal, $\lambda$ dependent exponent [29]. This is the so called Griffiths Phase (GP) [43, 44], bordered by a stretched exponential decay law at the critical point $\lambda_{c}^{0}$ of the clean system, and by the dirty critical point $\lambda_{c}>\lambda_{c}^{0}$, where the evolution becomes logarithmically slow

$$
\rho(t) \sim \ln \left(t / t_{0}\right)^{-\tilde{\alpha}},
$$

thanks to $\lim _{\lambda \rightarrow \lambda_{c}} b=0$. The $\tilde{\alpha}$ and other activated scaling exponents are described by the strong disorder universality fixed point in the renormalization sense. Extensive numerical simulations in [31] provided estimates for them in case of GSW-s.

\section{Griffiths phases in Generalized Small World network models}

GSW-s in one dimension have the intriguing feature that in the marginal case $(s=2)$ intrinsic properties exhibit power-law behavior and the corresponding exponents vary continuously with the prefactor $\beta$. First it was shown that the topological dimension of such networks (see Fig. 1a) depends on $\beta$ [46-48]. It has been claimed in a recent letter [29] and in [30, 31] that if $d(\beta)$ is finite, GP-s and similar rare-region effects can also appear. Density decay simulations, started from fully active state $(\rho(0)=1)$, have been presented in [31]. The effective decay exponents, defined as the local slope of $\rho(t)$ :

$$
\alpha_{\mathrm{eff}}(t)=-\frac{\ln \left[\rho(t) / \rho\left(t^{\prime}\right)\right]}{\ln \left(t / t^{\prime}\right)}
$$

saturate to $\lambda$ dependent constant values in the long time limit (Fig. 1b), although logarithmic corrections arise. 


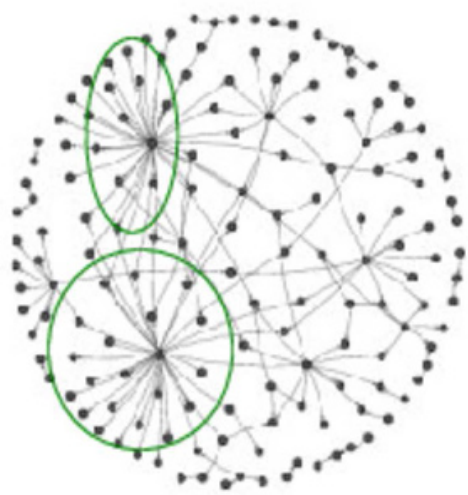

(a)

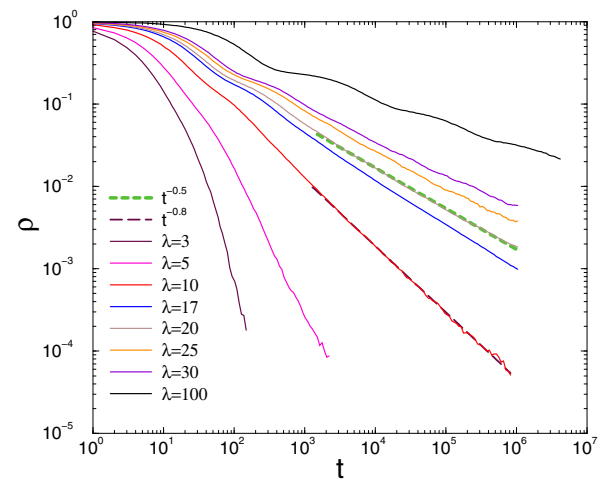

(b)

FIGURE 2: (a) Picture of an ER network exhibiting RR-s. (b) Density decay of the CP in ER below the percolation threshold. Dashed lines : power-law fits.

Interestingly such GP-s have also been found in 3-regular random networks, constructed as shown [49]. For larger values of $s$ the long-ranged links are irrelevant and $d(\beta)=1$, while for $s<2$ the topological dimension diverges and mean-field behavior emerges.

\section{Griffiths phases in Erdős Rényi network models}

The CP on Erdős Rényi (ER) graphs [51] with a quenched disordered infection rates (QCP) has been studied in [29-31]. A fraction $q$ of the nodes (type-II) propagate infection with a reduced value $\lambda r$, with $0 \leq r<1$, while the remaining fraction $1-q$ (type-I nodes) take their "clean" value $\lambda$. Pair mean-field approximations lead to the following critical threshold :

$$
\lambda_{c}(q)=\frac{\langle k\rangle}{\langle k\rangle-1} \frac{1}{1-q} .
$$

Type-I nodes experience a percolation transition, where the type I-to-type I average degree is 1, i.e. at $q_{\text {perc }}=1-\langle k\rangle^{-1}$. For $q>q_{\text {perc }}$ activity cannot be sustained : type-I clusters are finite and type-II ones do not propagate activity. Optimal fluctuation theory and simulations show that in this case the absorbing phase of QCP splits and a GP with power-law dynamics appears for $\lambda>\lambda_{c}^{0}$.

In fact in fragmented ER networks, where $d$ is zero the intrinsic disorder of $\lambda$-s is not necessary, but the topological heterogeneity itself is enough for the occurrence of GP-s as shown in Fig. 2b. Density decay simulations from fully active state of the CP on ER graphs with $\langle k\rangle=0.15$ up to sizes $N=10^{6}$ confirm this. For high values of $\lambda$ one can see plateaus on the $\rho(t)$ curves, similarly as reported in [27]. These are the consequence of the metastable local active domains.

\section{Slow dynamics in Barabási-Albert network models}

After showing the rare-region effects and the possibility of GP-s on GSW and ER networks let us turn towards the results on the most common type of scale-free (SF) networks. CP have been simulated 
and analyzed by mean-field methods on BA networks, in particular for loop-less and weighted cases as described in [45]. BA construction is a simple and fast way to generate SF networks, in contrast with other standard models, e.g [52]. The BA growth starts with a fully connected graph of small $\left(N_{0}=10\right)$ nodes. Following that at each generation step $s$, a new vertex with $m$ edges is added to the network and connected to an existing vertex $s^{\prime}$ of degree $k_{s^{\prime}}$ with probability $\Pi_{s \rightarrow s^{\prime}}=k_{s^{\prime}} / \sum_{s^{\prime \prime}<s} k_{s^{\prime \prime}}$. This process is iterated until reaching the desired network size $N$. The resulting network has a SF degree distribution $P(k) \simeq k^{-3}$ and for $m=1$ we obtain a BA tree (BAT) topology, while for the looped case $m=3$ is used. Binary (non-weighted) BA networks can be transformed into weighted ones by assigning to every edge connecting vertexes $i$ and $j$ a symmetric weight $\omega_{i j}$. In [45] two different network topology dependent weight assignment strategy was introduced in order to slow down and localize epidemics.

(i) Weighted BA tree I (WBAT-I) : Multiplicative weights, suppressing the infection capability of highly connected nodes

$$
\omega_{i j}=\omega_{0}\left(k_{i} k_{j}\right)^{-v}
$$

where $\omega_{0}$ is an arbitrary scale and $v$ is a characteristic exponent with $v \geq 0$. This can model internal limitations of hubs, like the sub-linear Heap's law [54].

(ii) Weighted BA tree II (WBAT-II) : Disassortatative weighting scheme according to the age of nodes in the network construction

$$
\omega_{i j}=\frac{|i-j|^{x}}{N}
$$

where the node numbers $i$ and $j$ correspond to the time step when they were connected the network. Since the degree of nodes decreases as $k_{i} \propto(N / i)^{1 / 2}$ during this process, this selection with $x>0$ favors connection between unlike nodes and suppresses interactions between similar ones.

The presence of these weights affects the dynamics of the CP. Thus, the rate at which a healthy vertex $i$ becomes ill on contact with an infected (active) vertex $j$ is proportional to $\lambda \omega_{i j}$, therefore the epidemic can in principle become trapped in isolated connected subsets. Density decay simulations [45], started from fully active state show GP like regions both in the WBAT-I and WBAT-II cases. For WBAT-II the scaling appears for $t>10^{4}$ MCs in the region $\lambda>9.5$, even in case of networks with $x=3$ as shown on Fig. 3a. The effective decay exponents, using $t / t^{\prime}=8$, saturate to $\lambda$ dependent constant values in the long time limit (see inset in Fig. 3a). However, by increasing $N$ at a given $\lambda$ the decay curves saturate asymptotically, suggesting a smeared phase transition as shown on Fig. $3 \mathrm{~b}$.

\section{Discussion and Conclusions}

I have overviewed the effects of quenched heterogeneity on the dynamics of the Contact Process in different network models. In finite topological dimensional cases slow dynamics (power-law, logarithmic, stretched exponential ... etc) and Griffiths Phases can be observed, which is very important for understanding spreading-like phenomena of real world networks. In these cases topological disorder of the network can result is GPs. Similarly, in models where the roles of space and time are exchanged "temporal Griffiths phases" have been found in various systems near their phase transition points [58, 59].

On the other hand on graphs of infinite topological dimensions like in weighted SF trees, the network heterogeneity can again cause slow dynamics of the CP. However, in the thermodynamic limit the power-laws saturate and the locally active, high dimensional subspaces cause smeared phase transitions [45]. Very recently slow dynamics has also been reported in case of the Susceptible-InfectedSusceptible model defined on the (SF) flower model [27], weigthed BA trees [28] and on ER graphs with exponential weights [60]. Spectral analysis of the quenched mean-field theory $[26,28]$ and simulations confirm the presence of epideimc localization effects in such models. Further studies of different models and networks are currently under way. 


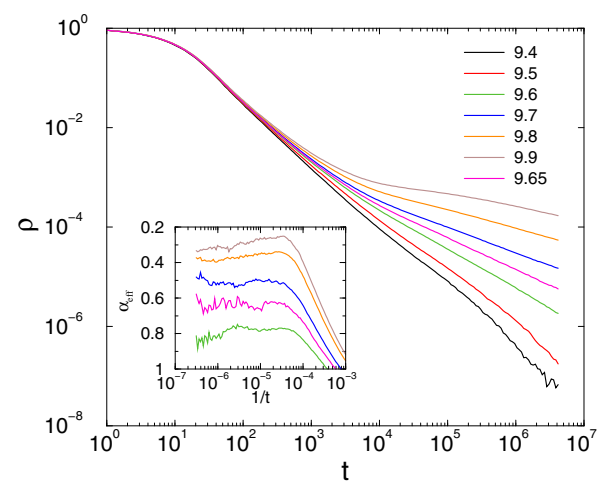

(a)

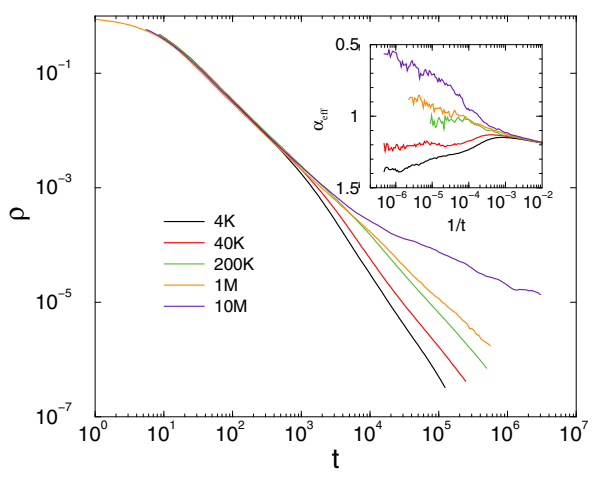

(b)

Figure 3: (a) Density decay as a function of time in CP defined on WBAT-II trees, with exponent $x=3$. Network size $: N=4 \times 10^{5}$. Different curves correspond to $: \lambda=9.4,9.5,9.6,9.65,9.7,9.8,9.9$ (from bottom to top). Inset : the corresponding local slopes (b) Density decay as a function of time in $\mathrm{CP}$ on a weighted WBAT-II trees with exponent $x=2$ at $\lambda=6.8$. Different curves correspond to sizes $N=4 \times 10^{3}, 4 \times 10^{4}, 2 \times 10^{5}, 10^{6}, 10^{7}$ (from bottom to top). Inset : the corresponding local slopes.

\section{Acknowledgments}

I thank R. Juhász and I. Kovács for their comments and acknowledge support from the Hungarian research fund OTKA (Grant No. T77629), HPC-EUROPA2 (pr. 228398) and the European Social Fund through project FuturICT.hu (grant no. : TAMOP-4.2.2.C-11/1/KONV-2012-0013).

\section{Références}

[1] J. Marro and R. Dickman, Nonequilibrium Phase Transitions in Lattice Models (Cambridge University Press, Cambridge, 1999) .

[2] G. Ódor, Universality in Nonequilibrium Lattice Systems (World Scientific, Singapore, 2008).

[3] M. Henkel, H. Hinrichsen, and S. Lübeck, Non-equilibrium phase transition : Absorbing Phase Transitions (Springer Verlag, Netherlands, 2008).

[4] G. Ódor, Rev. Mod. Phys. 76 (2004) 663.

[5] P. Bak, C. Tang and K. Wiesenfeld, Phys. Rev. Lett. 59, 381 (1987).

[6] T. E. Harris, Ann. Prob. 2, 969 (1974).

[7] T. M. Liggett, Interacting Particle Systems (Springer-Verlag, New York, 1985).

[8] R. Albert and A.-L. Barabási, Rev. Mod. Phys. 74, 47 (2002) .

[9] S. N. Dorogovtsev and J. F. F. Mendes, Evolution of networks : From biological nets to the Internet and $W W W$ (Oxford University Press, Oxford, 2003) .

[10] A.-L. Barabási and R. Albert, Science 286, 509 (1999) (Oxford University Press, Oxford, 1992).

[11] S. N. Dorogovtsev, A. V. Goltsev, and J. F. F. Mendes, Rev. Mod. Phys. 80, 1275 (2008).

[12] J. M. Kleinberg, Nature 406, 845 (2000).

[13] D. Chowdhury and B. Chakrabarti, J. Phys. A : Math. Gen. 18, L377 (1985).

[14] G. Li et al., Phys. Rev. Lett. 104, (2010) 018701. 
[15] J. Hicks, A. Benham and A. Ural, Phys. Rev E 79 (2009) 012102.

[16] D. Liben-Novell et al, PNAS 102, (2005) 11623.

[17] R. Lambiotte, et al, Physica A 387, 5317.

[18] L. Zekri et al. Phys. Lett. A 375 (2011) 346-351.

[19] G. Li et al, Phys. Rev. Lett 104, 018701 (2010)

[20] D. Mollison, J. R. Stat. Soc. B 39, (1977) 283.

[21] H. Hinrichsen, J. Stat. Mech. : Theor. Exp. P07066 (2007).

[22] A. Barrat, M. Barthélemy, and A. Vespignani, Dynamical Processes on Complex Networks (Cambridge University Press, Cambridge, 2008).

[23] C. Castellano and R. Pastor-Satorras, Phys. Rev. Lett. 96, 038701 (2006).

[24] C. Castellano and R. Pastor-Satorras, Phys. Rev. Lett. 100, 148701 (2008).

[25] M. Boguñá, C. Castellano, and R. Pastor-Satorras, Phys. Rev. E 79, 036110 (2009) .

[26] A. V. Goltsev, S. N. Dorogovtsev, J. G. Oliveira, and J. F. F. Mendes, Phys. Rev. Lett. 109, 128702 (2012)

[27] H. K. Lee, P.-S. Shim and J. D. Noh, arXiv :1211.2519.

[28] G. Ódor, Rare regions of the SIS model on Barabasi-Albert networks arXiv :1301.4407

[29] M. A. Muñoz, R. Juhász, C. Castellano, and G. Ódor, Phys. Rev. Lett. 105, 128701 (2010) .

[30] G. Ódor, R. Juhasz, C. Castellano, and M. A. Munoz, in Nonequilibrium Statistical Physics Today, Vol. 1332, edited by P. L. Garrido, J. Marro, and F. de los Santos (AIP, 2011) pp. 172178.

[31] R. Juhász, G. Ódor, C. Castellano, and M. A. Muñoz, Phys. Rev. E. 85, 066125 (2012) .

[32] S. Johnson, J. J. Torres, and J. Marro, PLoS ONE 8(1) : e50276 (2013).

[33] D. R. Chialvo, Criticality in Neural Systems, Niebur E, Plenz D, Schuster HG. (eds.) John Wiley $\mathcal{E}$ Sons (2013), arXiv :1210.3632

[34] X. Castelló, R. Toivonen, V. M. Eguíluz, J. Saramäki, K. Kaski and M. San Miguel, EPL 79 (2007) 66006.

[35] A. Amir, Y. Oreg, and Y. Imry, Phys. Rev. Lett. 105, 070601 (2010).

[36] A. J. Bray, Phys. Rev. Lett. 59, 586 (1987).

[37] D. Dhar, M. Randeria, and J. P. Sethna, Europhys. Lett. 5, 485 (1988).

[38] H. Rieger and A. P. Young, Phys. Rev. B 54, 3328 (1996).

[39] D. S. Fisher, Phys. Rev. Lett. 69, 534 (1992).

[40] C. Monthus, T. Garel, J. of Phys. A 44 (2011) 085001.

[41] I. A. Kovács, F. Iglói, J. Phys. : Condens. Matter 23 (2011) 404204.

[42] M. Karsai, et al. Phys. Rev. E. 83, 025102(R) (2011).

[43] R. B. Griffiths, Phys. Rev. Lett. 23, 17 (1969).

[44] T. Vojta, Journal of Physics A : Mathematical and General 39, R143 (2006).

[45] G.Ódor and R. Pastor-Satorras, Phys. Rev. E 86, (2012) 026117.

[46] I. Benjamini and N. Berger, Rand. Struct. Alg. 19, 102 (2001).

[47] D. Coppersmith, D. Gamarnik and M. Sviridenko, Rand. Struct. Alg. 21, 1 (2002).

[48] R. Juhász, Phys. Rev. E 85, 011118 (2012).

[49] R. Juhász, Phys. Rev. E 78, 066106 (2008).

[50] R. Juhász, G. Ódor, Phys. Rev. E 80, 041123 (2009).

[51] P. Erdős, A. Rényi, Publicationes Mathematicae 6, 290 (1959).

[52] M. Catanzaro, M. Boguñá, and R. Pastor-Satorras, Phys. Rev. E 71, 027103 (2005). 
[53] F. Chung, L. Lu and V. Vu, Proc. Natl. Acad. Sci. USA 1006313 (2003)

[54] H. Heaps, Information retrieval : Computational and theoretical aspects (Academic Press, Inc. Orlando, FL, USA, 1978).

[55] S. C. Ferreira, C. Castellano, R. Pastor-Satorras, Phys. Rev. E 86, 041125 (2012)

[56] M. Karsai, R. Juhász, and F. Iglói, Phys. Rev. E 73, 036116 (2006).

[57] G. Bianconi, J. Stat. Mech. (2012) P07021.

[58] F. Vazquez, J. A. Bonachela, C. Lopez, and M. A. Muñoz, Phys. Rev. Lett. 106, 235702 (2011).

[59] R. Martinez-Garcia, F. Vazquez, C. López and M. A. Muñoz, Phys. Rev E 85, 051125 (2012).

[60] C. Buono, F. Vazquez, P. A. Macri, L. A. Braunstein, arXiv :1301.1645v1. 